\title{
3rd generation SUSY searches at CMS
}

\author{
Alexis KALOGEROPOULOS, on behalf of the CMS collaboration [1]. \\ DESY
}

E-mail: alkaloge@cern.ch

\begin{abstract}
Supersymmetry (SUSY) is a very appealing theory which can help solving many of the shortcoming of the Standard Model (SM). Especially searches for $3^{\text {rd }}$ Generation sparticles are very well motivated due to the Naturalness. Indeed, if SUSY is realized this way, then the Higgs-boson mass can be stabilized without the need for fine-tuning. Further, $3^{r d}$ Generation squarks can be lighter than all squarks due to large mixing and below the TeV scale. In addition, the Wino, the Bino, the leptons and the rest of the squarks can have a mass $\mathscr{O}(\mathrm{TeV})$ with no further complications.
\end{abstract}

From experimental point of view, if $R$-parity is conserved, the SUSY particles have to be created in pairs, while the sparticles appear usually in long cascades which results in multi jet events with high $p_{\mathrm{T}}$ and/or the presence of leptons. Further, the Lightest Supersymmetry Particle (LSP) is stable resulting in $E_{\mathrm{T}}^{\prime}$ signatures in the detector. On the other hand if $R$-parity is not conserved in nature, then SUSY particles can be created singly while the LSP is not stable. The latter important phenomenological feature practically means that no additional $E_{\mathrm{T}}^{\prime}$ is created on the detector from the LSP.

In this note, some of the latest results from CMS for direct and indirect production of $3^{\text {rd }}$ Generation squarks in a variety of decay channels are reviewed. The results are based on the data collected during the $20128 \mathrm{TeV}$ LHC run. The full results can be found in the appended references and in [2].

XXII. International Workshop on Deep-Inelastic Scattering and Related Subjects

28 April - 2 May 2014

Warsaw, Poland 


\section{Search in opposite sign dilepton events, large number of jets, b-jets and $E_{\mathrm{T}}[11]$}

This analysis considers two opposite sign (OS) leptons, with at least two jets, b-tagged jets, and large $E_{\mathrm{T}}^{\prime}$ for $19.1 \mathrm{fb}^{-1}$ of integrated luminosity. The estimation of the SM background is completely data-driven while the results are interpreted in the context of SMS where pair produced gluinos decay to a pair of top quarks and a neutralino resulting to a final state with four top quarks.

\subsection{Event selection signal regions}

The baseline event selection includes two isolated OS leptons $(e e, \mu \mu$ or $e \mu)$ with $p_{\mathrm{T}}>20$ $\mathrm{GeV}$ and $|\eta|<2.4$, while at least two jets with $p_{\mathrm{T}}>30 \mathrm{GeV}$ and $|\eta|<2.4$ are required. Further, in order to maximize the sensitivity of the signal topology, signal regions (SR) are defined when $E_{\mathrm{T}}$ is above $180 \mathrm{GeV}$, while more that four jets are required with two of them being tagged as b-jets, while $\mid \eta<1$ for the two leading ones.

\subsection{Background estimation}

A Control Region (CR) is defined by inverting either one of the two signal selection criteria posed on the $\eta$ of the two leading jets. Next, an extrapolation factor is defined such as :

$$
R_{e x t}=\frac{N_{n b, S R}}{N_{n b, C R}}
$$

in bins of $E_{\mathrm{T}}$ for a given multiplicity on the b-jets. $R_{\text {ext }}$ features a smooth behavior as a function of the $E_{\mathrm{T}}$ which practically allows to extract the $R_{\text {ext }}$ directly from data. Combining the above information, the SM background prediction in the SR is then :

$$
N_{\text {Pred }}^{S R}=R_{\text {ext }}^{\text {Obs, nb-jets=2 }} \times N_{O b s}^{C R}
$$

as shown in Figure 1 (left).
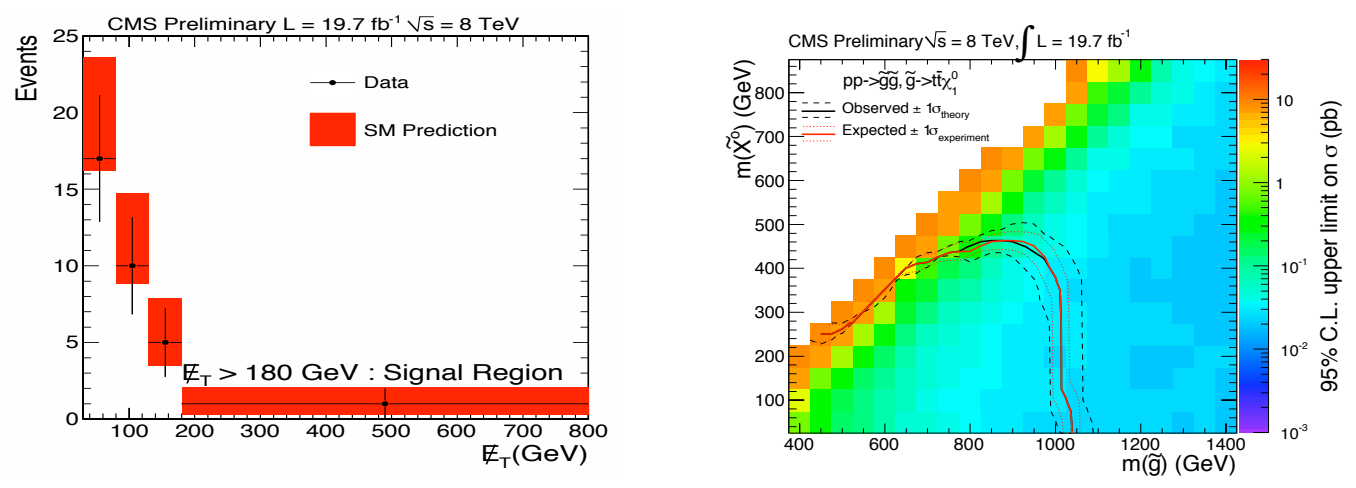

Figure 1: Left : The $N_{\text {Pred }}^{S R}$ for the $E_{\mathrm{T}}$. Signal region is defined when $E_{\mathrm{T}}>120 \mathrm{GeV}$. Right : The 95\% CL upper limits on the production cross-section the gluino decays according to $\tilde{g} \rightarrow \tilde{t} t \tilde{\chi}_{1}^{0}$.

\subsection{Results}

The results are interpreted on the SMS parameter space where upper limits on the production cross-section are set at 95\% CL. The exclusion on the considered SMS model is shown in Figure 1 (right) where $m_{\tilde{g}}$ masses around $1 \mathrm{TeV}$ are excluded for $m_{\tilde{\chi}_{1}^{0}}$ around $400 \mathrm{GeV}$. 


\section{Search for top-squark pair production in the single lepton final state [7]}

This analysis searches for stop quark pair production into two decay modes, namely $\tilde{t} \rightarrow t \tilde{\chi}_{1}^{0}$ and $\tilde{t} \rightarrow b \tilde{\chi}^{ \pm} \rightarrow b W \tilde{\chi}_{1}^{0}$ in a single lepton, large $E_{\mathrm{T}}$ and large transverse mass final state corresponding to $19.5 \mathrm{fb}^{1}$ of integrated luminosity. The main result is based on the BDT MVA method, while also a cut and count method is employed to serve as a cross check.

\subsection{Event selection and signal regions}

The event selection considers an isolated lepton $e(\mu)$ with $p_{\mathrm{T}}>30(25) \mathrm{GeV}$ within $|\eta|<$ 1.4442 (2.1). Further, at least four jets with $p_{\mathrm{T}}>30 \mathrm{GeV}$ and $|\eta|<2.4$ must be present with at least one being identified as a b-jet while $E_{\mathrm{T}}>100 \mathrm{GeV}$. The SR are defined when $M_{T}>120$ $\mathrm{GeV}$ and for the "cut-and-count" approach sequential cuts on individual variables are posed, while for the BDT MVA method, requirements are put on its final discriminant. The BDT is trained in several $\Delta M$ regions both for the signal and the background. The BDT output can be seen in Figure 2.

\subsection{Background estimation}

Top quark pairs are the dominant source of background when both the W-bosons decay leptonicaly. The rates are estimated from simulation and are further validated in control regions $(\mathrm{CR})$ where the background contributions are enriched. The $M_{T}$ distribution, especially in the $\mathrm{SR}(>120 \mathrm{GeV})$ is a critical variable in order to test the robustness of the method. Scale factors are also extracted from the CR to account for the discrepancies between data and prediction.

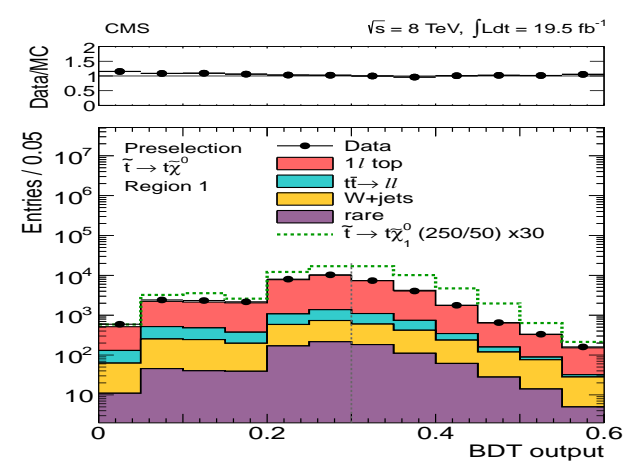

Figure 2: Data vs prediction for the BDT output.

\subsection{Results}

In the absence of significant excess, upper limits on the production cross section models have been set. The exclusion plots can be seen in Figure 3 where also the limits for different cases of the polarization of the top quark have been considered for the considered SMS models.

\section{Search for direct sbottom pairs production with $H_{\mathrm{T}}$ and $E_{\mathrm{T}}$ [12]}

This is a search for pair production of sbottom quarks decaying according to $\tilde{b} \rightarrow b \tilde{\chi}_{1}^{0}$ for a equivalent luminosity of $19.4 \mathrm{fb}-1$ of data. The analysis makes use of the of the boost-corrected contransverse mass defined as $m_{C T}=\sqrt{\left[E_{T}\left(J_{1}\right)+E_{T}\left(J_{2}\right)\right]^{2}-\left[\overrightarrow{p_{T}}\left(J_{1}\right)-\overrightarrow{p_{T}}\left(J_{2}\right)\right]^{2}}$ which has a characteristic endpoint defined by the $m_{\tilde{b}}$ and $m_{\tilde{\chi}_{1}^{0}}$. For a given jet $p_{\mathrm{T}}$, the bound of the distribution is saturated when the two jets point to the same direction in the transverse plane. 

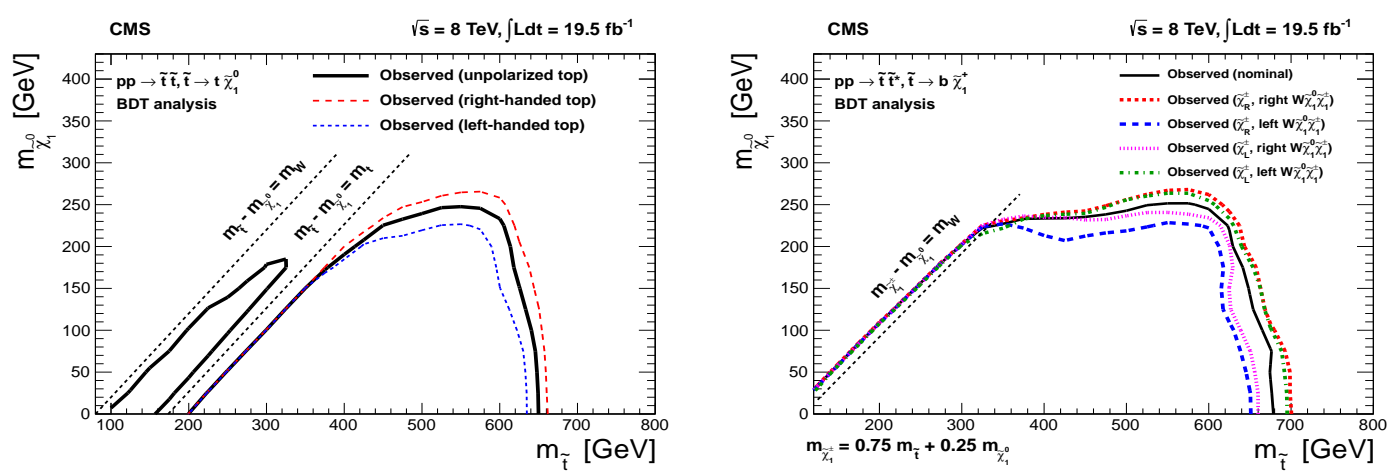

Figure 3: Exclusion plots at 95\% CL on the production cross-section for the $\tilde{t} \rightarrow t \tilde{\chi}_{1}^{0}$ (left) and the $\tilde{t} \rightarrow b \tilde{\chi}^{ \pm} \rightarrow b W \tilde{\chi}_{1}^{0}$ (right) SMS models.

\subsection{Event selection}

The baseline selection requires exactly two jets with $p_{\mathrm{T}}>70 \mathrm{GeV}$ within $|\eta|<2.4, \geq 1$ btagged jet and $H_{\mathrm{T}}>250 \mathrm{GeV}$. Signal enriched regions are defined by requiring $E_{\mathrm{T}}>175 \mathrm{GeV}$, $\Delta \phi\left(J_{1}, J_{2}\right)<2.5$ and $M_{T}\left(\mathbb{E}_{\mathrm{T}}, \mathrm{J}_{2}\right)>200 \mathrm{GeV}$. Further, events with an isolated lepton or track with $p_{\mathrm{T}}>10 \mathrm{GeV}$ are rejected so to suppress the background contributions. Figure 4 illustrates the $m_{C T}$ distributions after the baseline event selection with two signal points overlaid i.e. when $m_{\tilde{b}}=750$ (300) $\mathrm{GeV}$ and $m_{\tilde{\chi}_{1}^{0}}=50$ (150) GeV.

\subsection{Estimation of background}

$Z(v \bar{v})$ is the main background source, followed by the $W(\ell v)$ and $t \bar{t}$. To estimate them a single muon control sample is used in the signal regions. For the measurement of the $Z(v \bar{v})$, the events are required to not have any $b$-jets, while the $W(\ell v)$ and $t \bar{t}$ are measured using events satisfying the signal definition criteria but accepting isolated leptons as well. QCD contributions have been found to be negligible while also other background processes contribute at a few $\%$ level.

\subsection{Results}

Data was found to be consistent with the expectation, hence upper limits on the production cross section are set. The results are interpreted in the SMS model when the sbottom quark pair decays according to $\tilde{b} \rightarrow b \tilde{\chi}_{1}^{0}$. Sbottom masses up to $700 \mathrm{GeV}$ for neutralino masses less than $50 \mathrm{GeV}$ are excluded (Figure 5).

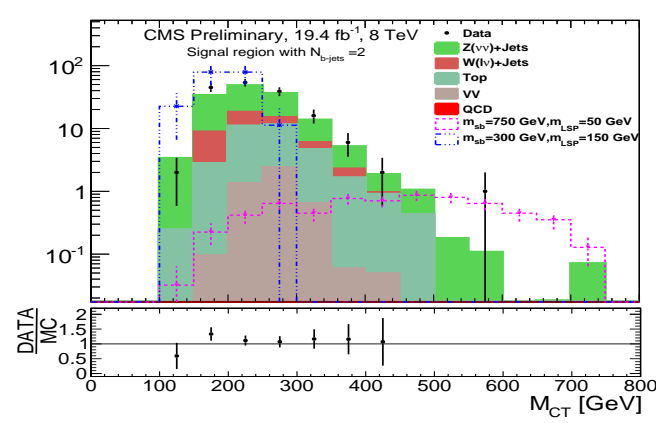

Figure 4: The $m_{C T}$ distributions after the baseline selection.

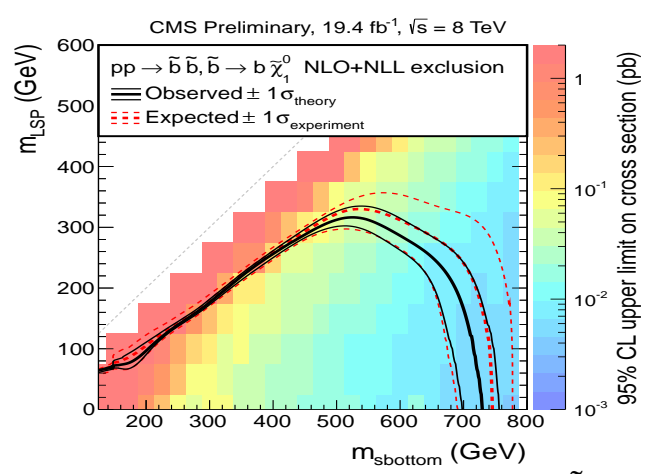

Figure 5: Exclusion plots at 95\% CL for $\tilde{b} \rightarrow b \tilde{\chi}_{1}^{0}$. 


\section{Search for top squarks decaying to a charm quark and a neutralino in events with a jet and $E_{\mathrm{T}}[6]$}

This analysis aims in specific decays of the stop quark according to $\tilde{t} \rightarrow c \tilde{\chi}_{1}^{0}$ when the mass splitting $\Delta M=m_{\tilde{t}_{1}}-m_{\tilde{\chi}_{1}^{0}}<80 \mathrm{GeV}$. The data sample corresponds to $19.7 \mathrm{fb}^{-} 1$ of int. luminosity.

\subsection{Events selection}

The baseline event selection requires the leading jet to have $p_{\mathrm{T}}>110 \mathrm{GeV}$ and $|\eta|<2.4$, while as signal events can contain both a jet from ISR or FSR, the 2nd leading jet is required to have $p_{\mathrm{T}}>$ $60 \mathrm{GeV}$ within $|\eta|<4.5$. This can keep the soft charm jets "invisible" while the QCD contribution is suppressed. Further, a veto on leptons is also posed, while $E_{\mathrm{T}}^{\prime}$ is required to be above $250 \mathrm{GeV}$. Several signal regions are defined depending on the $p_{\mathrm{T}}$ binning of the leading jet.

\subsection{Background estimation}

The dominant background processes are the $Z(v \bar{v})$ and the $W(\ell v)$. Both are estimated from data using a control sample of single muon events. The baseline selection is applied while the lepton veto is dropped. As the $Z(v \bar{v}) \& Z(\mu \bar{\mu})$ have similar kinematics, the former can be estimated:

$$
N_{Z(v \bar{v}}=\frac{N^{o b s}-N^{b g k d}}{A \times \varepsilon} \cdot R
$$

where $N^{o b s}$ is the number of dimuon events observed, $N^{b g k d}$ is the estimated number of background events, $A \& \varepsilon$ are the acceptance and selection efficiency, while $R$ is the ratio of the $Z(v \bar{v})$ and $Z(\mu \bar{\mu})$ BRs. The estimation of the $W(\ell v)$ contribution is evaluated from a $W(\mu v)$ control sample after requiring an isolated lepton with $p_{\mathrm{T}}>20 \mathrm{GeV}$ while the $50<M_{T}<100 \mathrm{GeV}$. The total events that are "lost", out of the acceptance or just misconstructed can be estimated :

$$
N_{\text {lost } \mu}=N_{\text {tot }} \times\left(1-A_{\mu} \varepsilon_{\mu}\right)
$$

where $A_{\mu} \& \varepsilon_{\mu}$ stands for the acceptance and the efficiency of the muon selection used in the lepton veto. A similar approach is used for the "lost" electrons as well. Finally, other background processes are estimated from simulation.

\subsection{Results}

No significant deviation from the expectation was observed and the results are interpreted in the context of the SMS model where a stop quark decays to a charm and a LSP and limits are set on the production cross section of the model. Further, the signal acceptance is greater for higher stop quark mass and small mass splitting, where the produced charm jets are softer. These events can be considered as "mono jet" like event as they are more boosted. Figure 6 shows the signal acceptances at $p_{\mathrm{T}}^{1}>300 \mathrm{GeV}$ for all of the considered mass points, as well as the upper limits on the production cross section of the SMS models. 

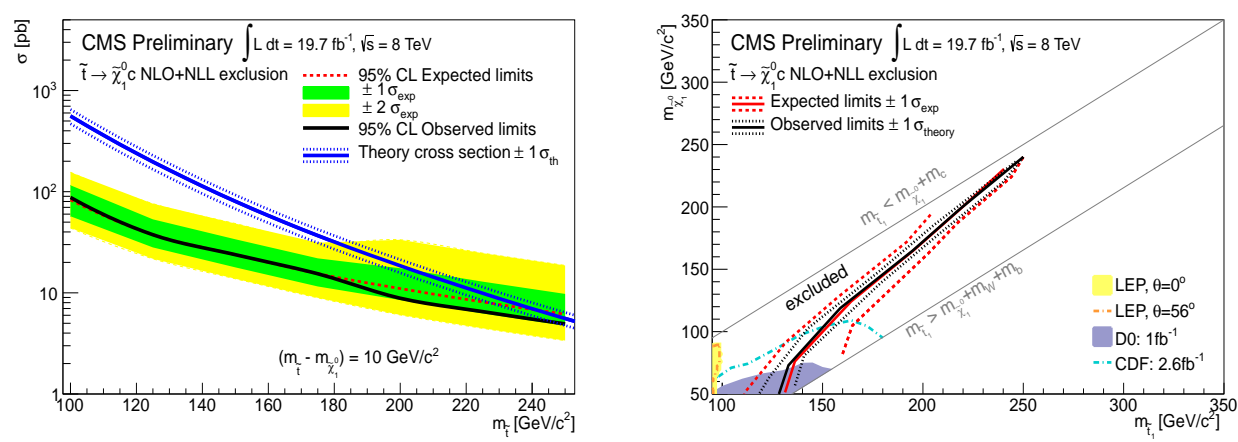

Figure 6: The signal acceptance at $p_{\mathrm{T}}^{1}>300 \mathrm{GeV}$ for all of the considered mass point (left) and the exclusion limits at $95 \%$ CL for sbottom quark pair production when sbottoms decay according to $\tilde{b} \rightarrow b \tilde{\chi}_{1}^{0}$. Sbottom masses up to $700 \mathrm{GeV}$ for neutralino masses less than $50 \mathrm{GeV}$ are excluded.

\section{References}

[1] The CMS experiment at the CERN LHC. doi : 10.1088/1748-0221/3/08/S08004 JINST, 3 S08004(2008)

[2] CMS SUSY Public results. https://twiki.cern.ch/twiki/bin/view/CMSPublic/PhysicsResultsSUS

[3] CMS Collaboration. Search for light stop RPV supersymmetry with three or more leptons and b-tags PRL 111, 221801 (2013)

[4] CMS Collaboration. Search for supersymmetry using razor variables in events with b-jets in $p p$ collisions at $8 \mathrm{TeV}$ CMS-PAS-SUS-13-004

[5] CMS Collaboration. Search for supersymmetry using events with a single lepton, multiple jets, and b-tags arXiv:1311.4937

[6] CMS Collaboration. Search for direct production of stops decaying to a charm and LSP using the monojet + MET final state CMS-PAS-SUS-13-009

[7] CMS Collaboration. Search for top-squark pair production in the single lepton final state in pp collisions at $8 \mathrm{TeV}$ EP JC 73 (2013) 2677

[8] CMS Collaboration. S Search for new physics in events with same-sign dileptons and jets in pp collisions at $8 \mathrm{TeV}$ JHEP 01 (2014) 163

[9] CMS Collaboration. Search for SUSY Partners of Top and Higgs Using Diphoton Higgs Decays in pp collisions at $8 \mathrm{TeV}$ CMS-PAS-SUS-13-015

[10] CMS Collaboration. Search for top squarks in multijet events with large missing momentum in pp collisions at $8 \mathrm{TeV}$ CMS-PAS-SUS-13-009

[11] CMS Collaboration. Search for SUSY in Opposite Sign Dilepton events, large number of jets, $b$-jets and MET in pp collisions at $8 \mathrm{TeV}$ CMS-PAS-SUS-13-016

[12] CMS Collaboration. Search for direct production of a pair of bottom squarks CMS-PAS-SUS-13-018

[13] CMS Collaboration. Search for supersymmetry using the shape of the HT and MET, and b-jet multiplicity distributions arXiv:1305.2390 